\title{
Sky coverage estimates for the natural guide star mode of the TMT facility AO system NFIRAOS
}

\section{B. Ellerbroek, David Andersen}

B. Ellerbroek, David Andersen, "Sky coverage estimates for the natural guide star mode of the TMT facility AO system NFIRAOS," Proc. SPIE 7015, Adaptive Optics Systems, 70155W (15 July 2008); doi: 10.1117/12.788053

SPIE Event: SPIE Astronomical Telescopes + Instrumentation, 2008, Marseille, France 


\title{
Sky coverage estimates for the natural guide star mode of the TMT facility AO system NFIRAOS
}

\author{
B. Ellerbroek ${ }^{a}$ and David Andersen ${ }^{b}$ \\ ${ }^{a}$ TMT Observatory Corporation, 2632 E. Washington Blvd, Pasadena, USA; \\ ${ }^{b}$ Herzberg Institute of Astrophysics, 5071 W. Saanich Road, Victoria, BC V9E 2E7, Canada
}

\begin{abstract}
Although the TMT AO system NFIRAOS will operate primarily in a laser guidestar multi-conjugate AO mode, it will also provide a conventional natural guide star (NGS) mode for use on very narrow science fields containing a bright star and/or when laser propagation is prevented by thin cirrus clouds or other circumstances. The number of bright stars suitable for use with a high order AO system is limited, so we have performed a sky coverage analysis to determine the likelyhood of achieving a given Strehl ratio when observing a randomly selected science field. The results obtained are significantly better than for existing NGS AO systems, largely due to (i) the anticipated availability of large, high-speed detector arrays with sub-electron read noise, and (ii) the benign telescope windshake disturbances predicted for TMT. Order $60 \times 60$ wavefront sensing and correction is preferred to lower order AO compensation, and an H-band Strehl of 0.25 [0.50] is obtained with sky coverage of about $1.0[0.1]$ per cent at the Galactic pole in median seeing. This level of performance will provide an important capability for TMT well into the life of the observatory.
\end{abstract}

Keywords: Thirty Meter Telescope, Adaptive Optics, NFIRAOS, Real Time Controller

\section{INTRODUCTION}

The TMT Science Requirements Document ${ }^{1}$ includes a basic requirement that the early light facility AO system NFIRAOS be operable with a single, bright natural guidestar. Although NFIRAOS will operate primarily in its laser guidestar multi-conjugate AO mode, this conventional natural guide star (NGS) mode is intended for use on very narrow science fields containing a bright star and/or when laser propagation is prevented due to thin cirrus clouds or other circumstances. This paper summarizes the results of an initial analysis undertaken during the last year to evaluate the potential sky coverage which might be obtained from NFIRAOS in NGS AO mode, and to commence the development of detailed requirements and design concepts derived from this level of performance.

The scientific utility of natural guidestar AO is significantly limited by anisoplanatism, which restricts observations to science targets which are quite close to relatively bright stars. This restriction is quantified in terms of a "sky coverage" function which relates the level of an NGS AO system's performance to the fraction of the sky over which it can be obtained. "Sky coverage" is a function of the design of the AO system, atmospheric turbulence conditions, the $\mathrm{AO}$ performance metric, and the density of guidestars in the direction of science observations (e.g., the galactic pole). A fairly standard model and set of expectations for NGS AO sky coverage was developed during the 1990's during the development of such systems for 8-10m class telescopes. ${ }^{3}$

During the last year, we have exercised this standard theory to evaluate sky coverage for the NFIRAOS NGS AO operating mode. The results obtained are dramatically superior to existing Shack-Hartmann-based NGS AO systems, but only if the NGS wavefront sensor is designed around a CCD array with sub-electron read noise. Subscale $128 \times 128$ and $160 \times 160$ arrays achieving this level of performance have now been demonstrated, ${ }^{4,5}$ and we intend to use a larger version of one of these devices for the NGS WFS instead of the "polar coordinate" AODP CCD array $^{6}$ as previously proposed. The resulting high-performance NGS AO capability will provide useful insurance against the possibilty that LGS MCAO commissioning activities may take longer than currently planned. The NGS AO mode will also continue to serve as a useful backup option during conditions of subvisible cirrus that prevent LGS AO operations, and also during (planned or unanticipated) laser downtime.

brente@caltech.edu; phone: 1626395 1620; www.tmt.org

Adaptive Optics Systems, edited by Norbert Hubin, Claire E. Max, Peter L. Wizinowich,

Proc. of SPIE Vol. 7015, 70155W, (2008)

0277-786X/08/\$18 $\cdot$ doi: $10.1117 / 12.788053$

Proc. of SPIE Vol. $701570155 \mathrm{~W}-1$ 
The remainder of this paper is organized as follows: Section 2 reviews our basic approach to estimating sky coverage, and section 3 summarizes all of the parameters and assumptions used in modeling the NFIRAOS NGS AO mode. Section 4 presents sample intermediate results from the calculations which illustrate the dramatic improvements in guide star limiting magnitude that can be achieved with very low read noise WFS detectors. The sky coverage results themselves are summarized in section 5 .

\section{ESTIMATING SKY COVERAGE}

The sky coverage of an AO system at a specified performance level $P_{*}$ (for example, a 50 per cent Strehl ratio in $\mathrm{J}$ band) is defined as the probability of surpassing that performance threshold for a randomly selected science target. Assuming that the occurance of guidestars within such a guidefield may be modeled as a Poisson process, this probability is given by

$$
\operatorname{Prob}\left(P \geq P_{*}\right)=1-\exp \left[-\bar{n}\left(P_{*}\right)\right],
$$

where $\bar{n}\left(P_{*}\right)$ is the expected number of guidestars which are sufficiently bright and sufficiently close to the science field to obtain performance level $P_{*}$. This quantity is in turn computed as

$$
\bar{n}\left(P_{*}\right)=\sum_{c} \int d m \rho(m ; c) \pi \theta^{2}\left(P_{*}, m ; c\right)
$$

where:

- $c$ is an index over guidestar spectral class;

- $m$ is the guidestar magnitude;

- $\rho(m ; c)$ is the differential density of guidestars of magnitude $m$ and spectral class $c$;

- $\theta\left(P_{*}, m ; c\right)$ is the allowable guide-star-to-science-target separation to achieve performance $P_{*}$ for a guidestar of magnitude $m$ and spectral class $c$.

We have gone to the additional trouble of parametrizing guide star densities and AO system performance as a function of guide star spectral class on account of the broad spectral bandpass planned for the NFIRAOS NGS wavefront sensor.

Evaluating the guide field diameter function $\theta(P, m ; c)$ from the results of an AO modeling code is essentially an inverse problem, since such simulations evaluate AO performance $p$ as a function of the guidestar offset $\vartheta$ and a number of other variables. These variables may be divided into:

- Atmospheric turbulence parameters $t$ which are fixed, independent of the choice of the guidestar;

- AO hardware parameters $h$ which are also fixed;

- AO control parameters $f$ which may adjusted to optimize performance (e.g., the WFS frame rate and the associated control loop bandwidth); and

- the RMS WFS subaperture tip/tilt measurement error $n$, which is generally a function of all of the above.

In the above notation, the determination of the field diameter $\theta$ takes the form

$$
\theta(P, m ; c)=\arg _{\vartheta}\left\{\max _{f} p[\vartheta, f, n(f, m ; c)]=P\right\},
$$

where we have suppressed the dependence of $p$ and $n$ upon the fixed parameters $t$ and $h$ for simplicity.

AO modeling codes which are both sufficiently accurate and computationally efficient enough to be used for computing the function $p$ above include PAOLA and CIBOLA (which has been used here). The theory of these codes is described elsewhere. ${ }^{7,8}$ In the remainder of this section we describe how we have computed the NGS 
WFS noise level $n$ as a function of $f, m$, and $c$ for the case of a Shack-Hartman WFS design based upon quadrant detectors, using a modest generalization of a classical result. ${ }^{9}$

In this case, the RMS WFS subaperture tip/tilt measurement error $n$ may be expressed as a ratio between an effective Shack-Hartmann spot radius and a subaperture signal to noise ratio, according to the formula:

$$
n(f, m ; c)=\frac{\theta_{B}(c)}{\operatorname{SNR}(f, m ; c)} .
$$

In the absence of sky background noise, the quadrant detector signal-to-noise ratio is given by the expression

$$
\operatorname{SNR}(f, m ; c)=\frac{N_{P D E}(m ; c) / f}{\sqrt{N_{P D E}(m ; c) / f+4 \sigma_{e}^{2}(f)}},
$$

where:

- $N_{P D E}(m ; c)$ is the number of photodetection events per WFS subaperture per second; and

- $\sigma_{e}(f)$ is the RMS detector read noise per pixel per frame, which may vary as a function of the frame rate $f$.

Next, the signal level $N_{P D E}$ may be computed as

$$
N_{P D E}(m ; c)=\exp (-m / 2.5) N_{0}(c),
$$

where the subaperture zeropoint $N_{0}(c)$ is given by the expressions

$$
\begin{aligned}
N_{0}(c) & =\int d \lambda r(\lambda ; c), \\
r(\lambda ; c) & =\tau_{a t m}(\lambda) \tau_{o p t}(\lambda) \eta(\lambda) n_{0}(\lambda ; c),
\end{aligned}
$$

and:

- $\lambda$ denotes wavelength within the NGS WFS spectral passband;

- $\tau_{\text {atm }}(\lambda)$ is the atmospheric transmittance function;

- $\tau_{\text {opt }}(\lambda)$ is the transmittance through the telescope and AO system optics to the NGS WFS focal plane;

- $\eta(\lambda)$ is the detector quantum efficiency; and

- $n_{0}(\lambda ; c)$ is the flux at the top of the atmosphere for an $m=0$ magnitude guidestar, measured in photons per second per subaperture per micron (of observing band).

Next, the broadband Shack-Hartmann spot radius $\theta_{B}(c)$ may be expressed as

$$
\theta_{B}(c)=\left[\frac{\int d \lambda \frac{r(\lambda ; c)}{\vartheta_{B}(\lambda)}}{\int d \lambda r(\lambda ; c)}\right]^{-1},
$$

where $\vartheta_{B}(\lambda)$ is the monochromatic spot radius at wavelength $\lambda$. Finally, the monochromatic short-exposure (i.e., tip-tilt removed) spot radius may be computed using the formulas

$$
\begin{aligned}
\vartheta(\lambda) & =\left\{4\left(d_{s} / \lambda\right) \int_{0}^{1} d \kappa \mathrm{OTF}\left[\left(\left(d_{s} / \lambda\right) \kappa, 0\right) ; \lambda\right]\right\}^{-1} \\
\mathrm{OTF}[(d / \lambda) \vec{\kappa} ; \lambda[ & =\int d \vec{r} A(\vec{r}) A(\vec{r}-d \vec{\kappa}) \exp \left[-\left(2 \pi^{2} / \lambda^{2}\right) D_{o p d}(\vec{r}, d \vec{\kappa})\right] \\
D_{\text {opd }}(\vec{r}, \vec{\delta}) & =\left\langle[\operatorname{OPD}(\vec{r})-\operatorname{OPD}(\vec{r}+\vec{\delta})]^{2}\right\rangle,
\end{aligned}
$$

where $d_{s}$ is the width of the subaperture, OTF is the optical transfer function for the short-exposure subaperture $\mathrm{PSF}$, and OPD is the tip/tilt-removed turbulence-induced optical path difference profile within the subaperture. 


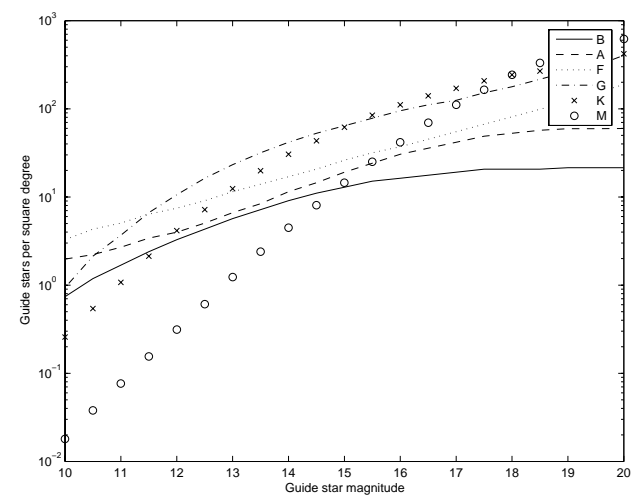

Figure 1. Guidestar density functions, derived from the Bahcall-Soneira model at the galactic pole.

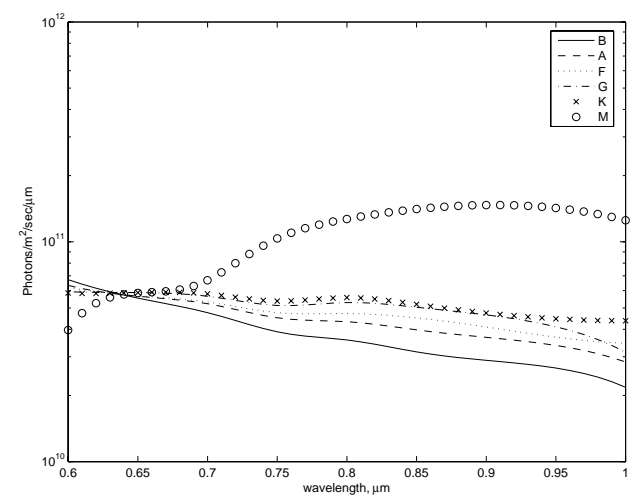

Figure 2. Guidestar spectral radiance functions at the top of the atmosphere for $\mathrm{R}=0$ magnitude stars.

\section{INPUT PARAMETER SUMMARY}

Sky coverage calculations require a range of input parameters and modeling assumptions related to the natural guidestars, the atmosphere, the telescope, the AO hardware and control system, and the performance evaluation metrics to be considered. These quantities are outlined in the following subsections.

\subsection{Guidestar Parameters}

The guidestar density model used in this work was obtained by decomposing the Bahcall-Soneira model for the galactic pole into separate density functions for B, A, F, G, K, and M class stars. ${ }^{3,10}$ Figure 1 plots the integrated guide star density functions for the number of guidestars brighter than a given magnitude in each of these six spectral classes.

The reason for introducing this additional complexity are the large differences in the shape of the spectral radiance function between "hot" and "cool" stars. We have smoothed and rebinned data from the STELIB library ${ }^{11}$ to derive the spectral radiance functions that we use in this analysis. Figure 2 plots the spectral radiance functions (evaluated at the top of the atmosphere) for $\mathrm{B}$ through $\mathrm{M}$ class stars at magnitude $\mathrm{R}=0$.

\subsection{Atmospheric Parameters}

Figure 3 plots the atmospheric optical transmittance in the spectral passband of the NGS WFS.

The atmospheric turbulence and windspeed profiles used in this analysis are summarized in Table 1 below. We have considered a pair of 7-layer $C_{n}^{2}$ profiles corresponding to median and 25 per cent seeing at Armazones, as described in the June 2007 edition of the TMT site survey report. ${ }^{13}$ Tony Travouillon has generated a preliminary 


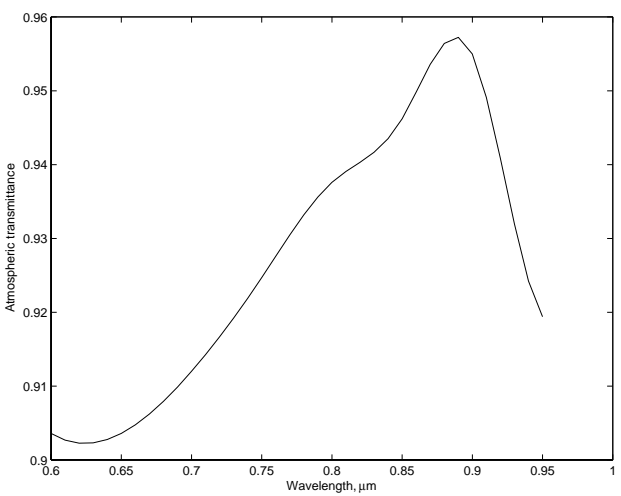

Figure 3. Atmospheric transmittance function in the NFIRAOS NGS WFS spectral passband.

median wind velocity profile by projecting thermosonde wind measurements from the Antofagasta airport onto the 7 overlapping altitude "bins" of the MASS/DIMM turbulence measurements used to generate these profiles. Finally, the associated values of the atmospheric turbulence parameters $r_{0}, \theta_{0}$, and $f_{g}$ are listed in Table 2 for the median and 25 per cent turbulence profiles.

Table 1. Atmospheric profile summary

\begin{tabular}{|c|cccc|}
\hline Layer number & Altitude, $\mathrm{km}$ & \multicolumn{2}{|c|}{ Fractional $C_{n}^{2}$} & Windspeed, m/s \\
& & $50 \%$ Seeing & $25 \%$ Seeing & \\
\hline 1 & 0.0 & 0.538 & 0.569 & 5.47 \\
2 & 0.5 & 0.052 & 0.021 & 5.92 \\
3 & 1.0 & 0.009 & 0.006 & 6.21 \\
4 & 2.0 & 0.047 & 0.022 & 7.63 \\
5 & 4.0 & 0.087 & 0.083 & 11.52 \\
6 & 8.0 & 0.144 & 0.142 & 24.99 \\
7 & 16.0 & 0.124 & 0.158 & 11.01 \\
\hline
\end{tabular}

Table 2. Atmospheric turbulence parameter summary

\begin{tabular}{|c|cc|}
\hline Parameter & $50 \%$ profile value & $25 \%$ profile value \\
\hline$r_{0}, \mathrm{~m}$ & 0.161 & 0.206 \\
$\theta_{0}$, arc sec & 1.832 & 2.118 \\
$f_{g}, \mathrm{~Hz}$ & 29.047 & 22.791 \\
\hline
\end{tabular}

\subsection{Telescope Parameters}

The TMT clear aperture has been approximated as circular and unobscured, with a diameter of 30 meters. The optical throughput is estimated to be 0.91 across the NGS WFS spectral passband. Uncorrectable higher-order telescope wavefront errors have been included as part of an overall implementation error budget as described further in section 3.6 below. Telescope tip/tilt jitter due to windshake has been neglected for the initial sky coverage analysis, although (as described further in section 4) we believe that the residual windshake jitter will be a small fraction of the uncorrected atmospheric turbulence errors for either bright or faint natural guide stars. 


\subsection{AO Hardware Parameters}

The only real AO "hardware" parameters modeled in CIBOLA for a classical NGS AO system are (i) the order of wavefront sensing and correction, and (ii) the magnitude of the wavefront sensor measurement noise. For item (i), a pair of NGS AO system options with order $60 \times 60$ and $30 \times 30$ wavefront sensors have been considered. For (ii), the WFS measurement noise level have been computed using the noise model presented in section 2, the radiometric parameters outlined in subsections 3.1 through 3.3 above, and the AO throughput and WFS CCD parameters summarized in the following paragraphs.

The AO system optical throughput up to (but not including) the NGS WFS CCD is estimated to be 0.62, yielding an end-to-end throughput of 0.56 when the telescope optics are also included. The NGS WFS samples 100 per cent of these photons in the passband between 0.63 and $0.95 \mu \mathrm{m}$.

Two different sets of WFS CCD array parameters have been evaluated, based upon extrapolations of the E2V CCD-60 and the MIT/LL CCID-56b detectors to larger arrays with $240 \times 240$ pixels.* Both of these arrays provide high quantum efficiency with low detector read noise at high frame rates, and the final sky coverage estimates for these two options are remarkably similar.

Figure 4 plots the quantum efficiency estimates used for these two devices. Both CCDs provide good QE across the full NFIRAOS NGS WFS spectral passband, but it should be noted that the E2V detector effectively suffers a further factor of two reduction in QE due to the so-called "excess noise" in the high-gain readout process. Table 3 lists the resulting radiometric zeropoints for the 6 guidestar spectral classes with these two quantum efficiency functions, taking into account all of other throughput losses associated with the atmosphere and optical train.

Table 3. Guidestar zeropoints for the NFIRAOS NGS WFS spectral passband

\begin{tabular}{|ccc|}
\hline Guide star class & \multicolumn{2}{c|}{ Zeropoint, PDE's/sec/m ${ }^{2}$} \\
& E2V CCD-60 & MIT/LL CCID-56b \\
\hline B & $2.77 \mathrm{e} 9$ & $5.69 \mathrm{e} 9$ \\
$\mathrm{~A}$ & $3.18 \mathrm{e} 9$ & $6.57 \mathrm{e} 9$ \\
$\mathrm{~F}$ & $3.38 \mathrm{e} 9$ & $6.99 \mathrm{e} 9$ \\
$\mathrm{G}$ & $3.68 \mathrm{e} 9$ & $7.62 \mathrm{e} 9$ \\
$\mathrm{~K}$ & $3.79 \mathrm{e} 9$ & $7.86 \mathrm{e} 9$ \\
$\mathrm{M}$ & $7.48 \mathrm{e} 9$ & $1.59 \mathrm{e} 10$ \\
\hline
\end{tabular}

The detector read noise for the CCD-60 is exceptionally low (about 0.25 electrons/pixel/read), even at a full-frame readout time of about $500 \mu \mathrm{sec}$ and frame rates of up to $1500 \mathrm{~Hz}$. This readout time is small enough that we have treated it as negligible in modeling the temporal dynamics of the AO control loop, although this may be slightly optimistic at the very highest WFS frame rates considered. The detector read noise for the CCID-56b is somewhat higher in comparison (see figure 5) and ranges from 0.6 to 1.6 electrons/pixel/read for frame rates between about 50 to $1500 \mathrm{~Hz}$. These results assume that the pixel digization rate has been minimized by setting the CCD readout time equal to the inverse of the frame rate. This additional latency impacts AO control loop dynamics as described in subsection 3.5 below.

Finally, although CIBOLA neglects AO implementation error sources such as DM hysteresis or DM/WFS misregistration, current estimates of the implementation errors from the NFIRAOS wavefront error budget have been included in the performance estimates as summarized in section 3.6.

\footnotetext{
${ }^{*}$ Although it is true that only $120 \times 120$ pixels would be required for an order $60 \times 60$ WFS with $2 \times 2$ pixels/subaperture, a more generous $4 \times 4$ pixels/subaperture is generally preferred to improve either the WFS FoV and/or the spatial sampling of the Shack-Hartmann spots.
} 


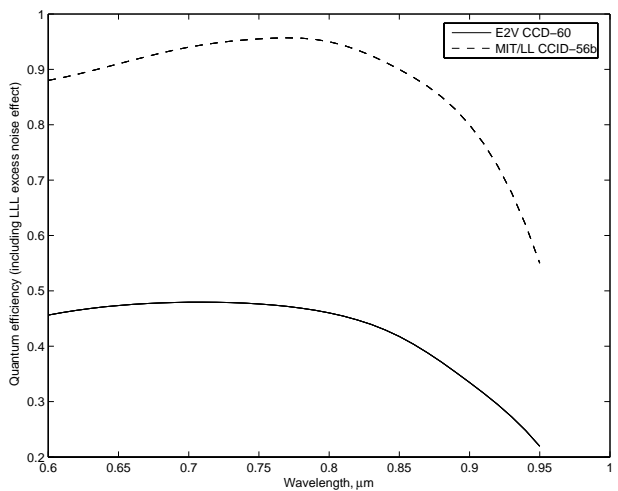

Figure 4. Quantum efficiency estimates for two candidate NGS WFS CCD arrays.

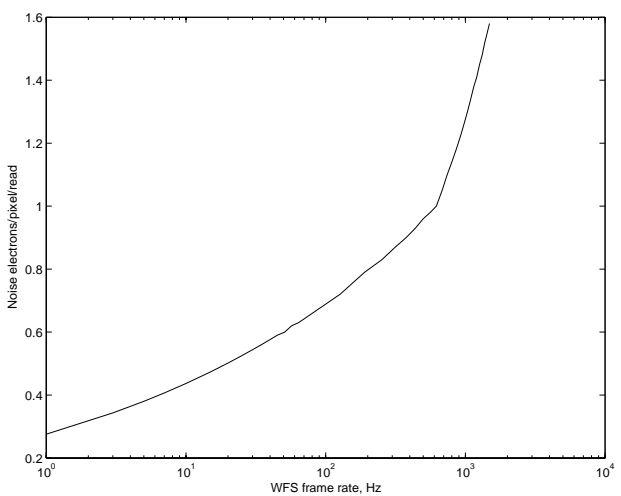

Figure 5. Detector read noise for the MIT/LL CCID-56b array with the detector readout time equal to inverse of the WFS frame rate. The values below $50 \mathrm{~Hz}$ have been extrapolated.

\subsection{AO Control Parameters}

The control parameters for a classical NGS AO system include the choice of the wavefront reconstruction (or estimation) matrix and the servo loop compensation parameters. Both of these quantities may be tuned to optimize AO system performance for a given WFS signal level and set of operating conditions.

The CIBOLA code uses a spatial frequency domain model for wavefront reconstruction and control. Because aperture edge effects are neglected, each Fourier mode of the wavefront within the spatial passband of the DM is reconstructed from the corresponding spatial frequency of the $x$ - and $y$-gradient measurements from the NGS WFS. For the current sky coverage analysis, the coefficients for each of these many $2 \times 1$-dimensional wavefront reconstruction problems were selected to minimize the residual wavefront error given a priori knowledge of the atmospheric parameters, WFS noise level, and the (global) control loop bandwidth, subject to the constraint that static wavefront errors must be fully corrected. Since all wavefront modes are controlled at a common temporal bandwidth, this last constraint implies that the wavefront variance in high spatial frequency modes may be amplified for the case of a high control bandwidth and a faint NGS. Such modes have been dropped from the wavefront reconstruction matrix and left uncontrolled for the sake of obtaining AO performance estimates.

A fairly modest (but still frustrating) approximation is introduced by the fact that while real-world AO systems operate as sampled data systems with control laws defined by discrete, digital filters, the only control law modeled by CIBOLA is an idealized, continuous-time, type I servo described by the formula

$$
\frac{d c}{d t}=-2 \pi f e(t)
$$


where $c(t)$ is the DM actuator command at time $t, e(t)$ is the reconstructed wavefront error expressed in terms of actuator influence functions, and $f$ is the (-3dB error rejection) bandwidth of the servo. Scaling factors must therefore be determined to relate the bandwidth $f$ to the WFS frame rate, and to relate the RMS error in $c(t)$ due to the propagation of WFS measurement noise to the corresponding error in $e(t)$. For the E2V LLL CCD array, the readout time of the detector is so short that we have assumed a digital control law of the form

$$
c(m)=c(m-1)+\frac{1}{2} e(m-1),
$$

where $m$ is a discrete time variable. In this case, the ratio between the WFS frame rate and the control loop bandwidth is about $15-1$, and the RMS error in $c$ due to the WFS measurement noise is $1 / \sqrt{3}$ times the RMS noise in $e$. Note that although the control loop bandwidth could be increased by replacing the gain coefficient $\frac{1}{2}$ with a larger value, the noise in $c$ would increase more rapidly. The preferred approach to increasing gain is to increase the WFS frame rate, particularly for a low read noise detector such as the E2V LLL CCD.

For the CCID-56b array, we have assumed that the CCD array is read out as slowly as possible (i.e., over a full WFS integration frame time) to minimize the WFS measurement noise. This adds an additional cycle of latency to the AO control loop, and the digital control law now becomes

$$
c(m)=\frac{1}{2} c(m-1)+\frac{1}{2} c(m-2)+\frac{1}{2} e(m-2) .
$$

The ratio between the WFS frame rate and the control loop bandwidth is now about 25-1, and the ratio between the RMS noise in $c$ and $e$ remains the same at a factor of $1 / \sqrt{3}$.

\subsection{Performance Metrics}

Only on-axis AO system performance has been evaluated. The performance metrics considered include: The RMS residual optical path difference, the long-exposure Strehl ratio at wavelength of $1.25,1.65$, and $2.2 \mu \mathrm{m}$ (referred to as $\mathrm{J}, \mathrm{H}$, and $\mathrm{K}$ bands below), and the long-exposure enclosed energy in a square, 50 milli-arc-second pixel at the same wavelengths. Based upon the current NFIRAOS wavefront error budget, ${ }^{12}$ an additional RMS OPD of $101 \mathrm{~nm}$ due to implementation error sources (telescope, NFIRAOS, and science instrument) has been included in all performance estimates unless stated otherwise. As a worst-case (but probably fairly accurate) assumption, this error reduces the delivered Strehl ratio and the 50 mas included energy values according to the Marechal approximation, with the lost energy scattered into the much larger "halo" of the partially-corrected AO PSF.

\section{GUIDESTAR LIMITING MAGNITUDES}

In addition to sky coverage, a second metric for quantifying the "reach" of a NGS AO system is the guidestar limiting magnitude associated with a given level of on-axis performance. The on-axis performance of the AO system is optimized by selecting control parameters which minimize the combined impact of WFS measurement noise, servo lag, and fitting error, i.e.,

$$
P_{0}(m ; c)=\max _{f} p[\vartheta=0, f, n(f, m ; c)]
$$

in the notation of section 2. The limiting magnitude of an NGS AO system is a useful indication of the system's utility for observing bright, point-like science targets which are themselves usable as guidestars. It also provides an initial indication of whether sky coverage will be high or low.

Figure 6 plots the on-axis Strehl ratios achieved by the NFIRAOS NGS AO system in J, H, and K bands as a function of the guidestar R-band magnitude with detector noise levels of 0.25 and 5 noise electrons per pixel per read. These results assume order $60 \times 60$ wavefront compensation, the E2V CCD-60 WFS detector parameters (apart from the case of 5 noise electrons), median seeing, a class $\mathrm{F}$ guidestar, and the remainder of the simulation parameters summarized in section 3 above. ${ }^{\dagger}$ Not surprisingly, detector read noise has a very dramatic impact upon $\mathrm{AO}$ system performance with faint guidestars. For example, the $\mathrm{H}$ band Strehl ratio equals 0.41 (one-half

\footnotetext{
${ }^{\dagger}$ Note that the NFIRAOS implementation error budget of $101 \mathrm{~nm}$ RMS is not included in these Strehls.
} 


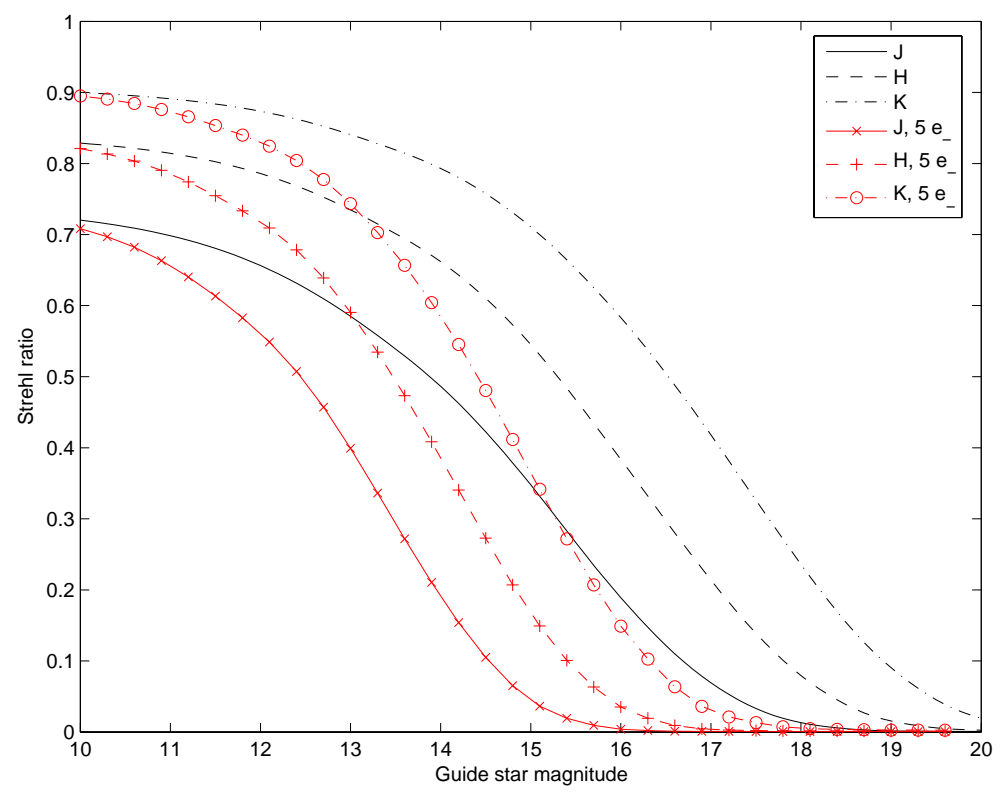

Figure 6. On-axis Strehl ratios vs guidestar R-band magnitude for the case of order $60 \times 60$ wavefront compensation, median seeing, E2V CCD-60 detector parameters, and a class F guidestar. Performance with 5 noise electrons per pixel per read is shown for the sake of comparison.

of the bright star limit) for a guidestar magnitude of about $\mathrm{R}=16$ [14] in the case of 0.25 [5] noise electrons, and similar improvements are obtained in $\mathrm{J}$ and $\mathrm{K}$ bands as well. Very useful levels of AO compensation are achieved in $\mathrm{H}$ and $\mathrm{K}$ bands with guidestars as faint as 18th magnitude in R-band. On account of these results, we propose to substitute one of the low-read-noise CCD options described in this memo for the "polar coordinate" AODP CCD array originally proposed for the NFIRAOS NGS WFS, which we expect to have 3 (or more) read noise electrons on account of much higher pixel read rates.

Figures 7 through 10 present the values of several intermediate quantities which may help to illustrate how these levels of performance are achieved. Figure 7 plots the optimum control loop bandwidth as a function of guidestar R-band magnitude, which declines from about 46 to $1.5 \mathrm{~Hz}$ as the guidestar magnitude increases from $\mathrm{R}=10$ to 20 . Figure 8 plots the corresponding WFS signal level for a WFS frame rate equal to 15 times the AO control loop bandwidth, and figure 9 plots the resulting WFS subaperture SNR at the control loop bandwidth (i.e., scaled by the noise attentuation factor of $1 / \sqrt{3}$ corresponding to the digital filter given in Eq. (14)). Finally, figure 10 plots the RMS phase difference measurement error across the $0.5 \mathrm{~m}$ width of a WFS subaperture. More detailed modeling is probably needed to confirm these results for guidestars dimmer than about 18th magnitude in R-band, since at this point the WFS signal level drops below one PDE per subaperture per frame. Still, the significance of sub-electron read noise for wavefront sensing with faint NGS is very apparent.

Finally, we need to note that neither sky background or telescope windshake has been considered in this analysis. The former effect is unlikely to be significant for guidestars brighter than about magnitude $\mathrm{R}=18$, since the optical sky background at dark sites is typically no greater than about magnitude 19 per square arc second. For example, the 50\% [80\%] R-band sky background at Mauna Kea is about 19.9 [19.2] magnitudes per square arc second; ${ }^{14}$ this corresponds to an SNR reduction of about $8 \%$ [13\%] for a magnitude $\mathrm{R}=18$ guidestar and a typical WFS subaperture FoV of one arc second.

Telescope windshake also appears to be a virtually negligible effect on account of the benign tip/tilt jitter PSD now predicted for the TMT telescope structure. ${ }^{15}$ For example, a residual tip/tilt jitter of only $5 \mathrm{~nm}$ RMS (or 0.143 mas) is predicted with a WFS frame rate of $90 \mathrm{~Hz}$ using the NFIRAOS "woofer-tweeter" tip/tilt control architecture. ${ }^{16}$ This frame rate corresponds to an AO control loop bandwidth of about $6 \mathrm{~Hz}$, which (according to figure 7) can be supported with a guidestar of magnitude 17 in R-band. 


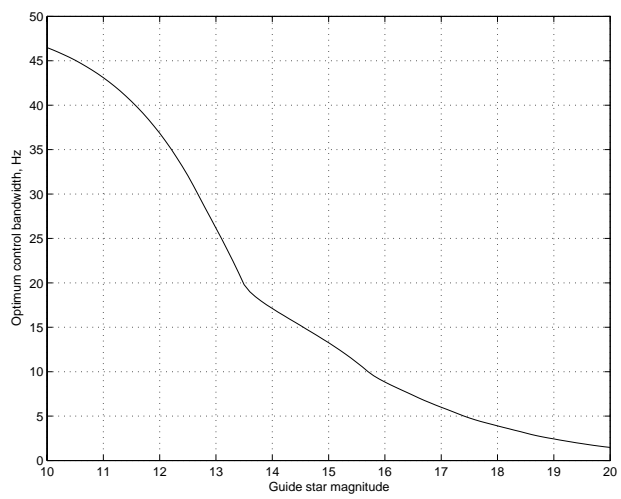

Figure 7. Optimized control bandwidths vs guidestar R-band magnitude for the case of order $60 \times 60$ wavefront compensation, median seeing, E2V CCD-60 detector parameters, and a class F guidestar.

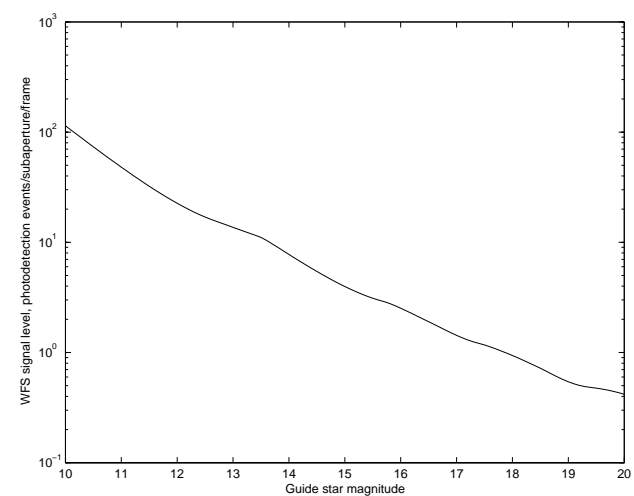

Figure 8. WFS signal level vs guidestar R-band magnitude for the case of order $60 \times 60$ wavefront compensation, median seeing, E2V CCD-60 detector parameters, and a class F guidestar.

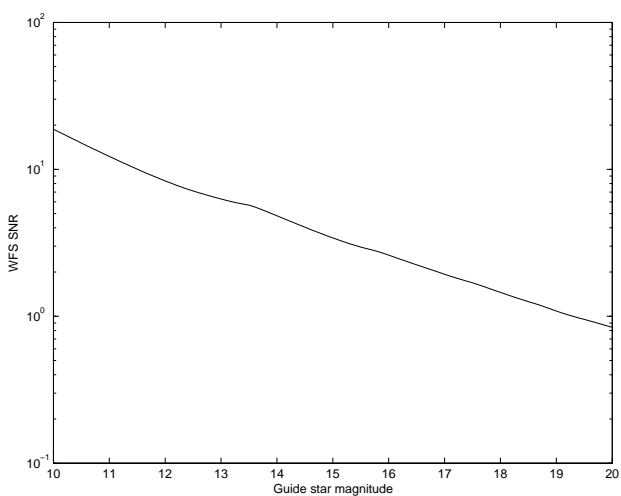

Figure 9. WFS signal-to-noise ratio vs guidestar R-band magnitude for the case of order $60 \times 60$ wavefront compensation, median seeing, E2V CCD-60 detector parameters, and a class F guidestar. 


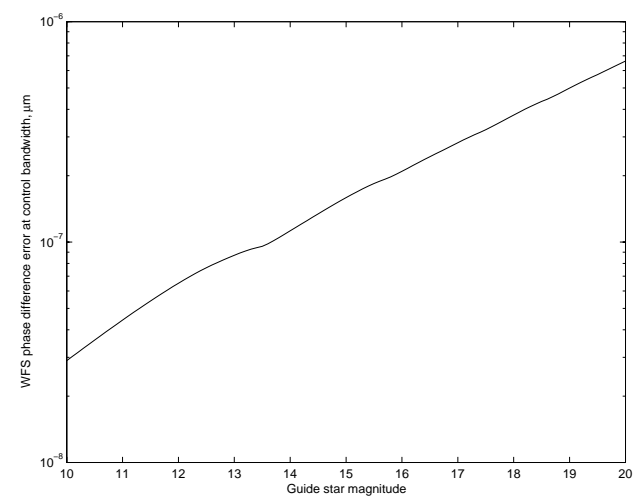

Figure 10. WFS RMS phase difference measurement error vs guidestar R-band magnitude for the case of order $60 \times 60$ wavefront compensation, median seeing, E2V CCD-60 detector parameters, and a class F guidestar.

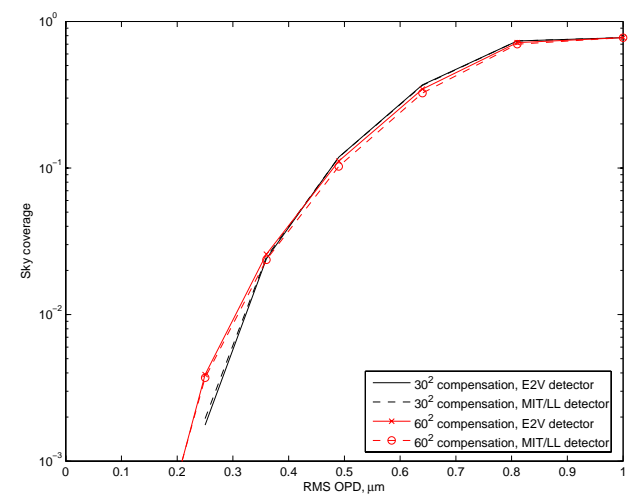

Figure 11. Sky coverage as a function of the residual RMS OPD for the NFIRAOS NGS AO system. These results are for the case of median seeing, and the NFIRAOS implementation error budget of $101 \mathrm{~nm}$ RMS is not included.

\section{SKY COVERAGE SUMMARY}

Figure 11 plots sky coverage as a function of the residual RMS OPD for the NFIRAOS NGS AO system. These results are for the case of median seeing, and the NFIRAOS implementation error budget of $101 \mathrm{~nm}$ RMS is not included. Systems with either $30 \times 30$ or $60 \times 60$ wavefront compensation have been considered, together with either CCD-60 or CCID-56b performance characteristics for the NGS WFS detector array. This yields a total of 4 different system configurations, all of which yield fairly similar performance. The performance achieved with the two different detector arrays is virtually identical, presumably because (i) both detectors have very low read noise and (ii) the reduced control loop latency provided by the CCD-60 compensates for the "excess noise" in the avalance readout process. As expected, the sky coverage obtained with $60 \times 60$ wavefront compensation is superior at very high performance levels due to the reduced fitting error, while a $30 \times 30$ system is preferred at low performance levels on account of the reduction in overall WFS measurement noise with fewer subapertures. This latter advantage is very modest because of the very low readout noise provided by either of the two detector options.

Figure 12 plots the relative sky coverage obtained with the four different sets of AO hardware parameters to highlight the fairly small differences in their performance. Based upon these results, we conclude that (i) order $60 \times 60$ wavefront compensation is preferred, and that (ii) the performance obtained with the two different WFS detector options is effectively equivalent, at least at this level of modeling accuracy. We will restrict attention to an order $60 \times 60$ system using the $\mathrm{E} 2 \mathrm{~V}$ detector parameters for the remainder of this section.

Next, figure 13 compares sky coverage as a function of the residual RMS OPD under median and $25 \%$ seeing 


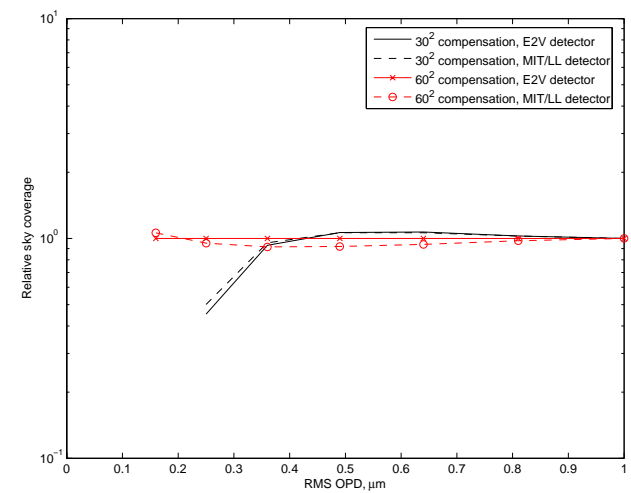

Figure 12. Relative sky coverage as a function of the residual RMS OPD for the 4 different NGS AO configurations considered in Figure 11 above. Ratios are computed relative to the sky coverage obtained with order $60 \times 60$ wavefront compensation and the E2V CCD-60 WFS detector parameters.

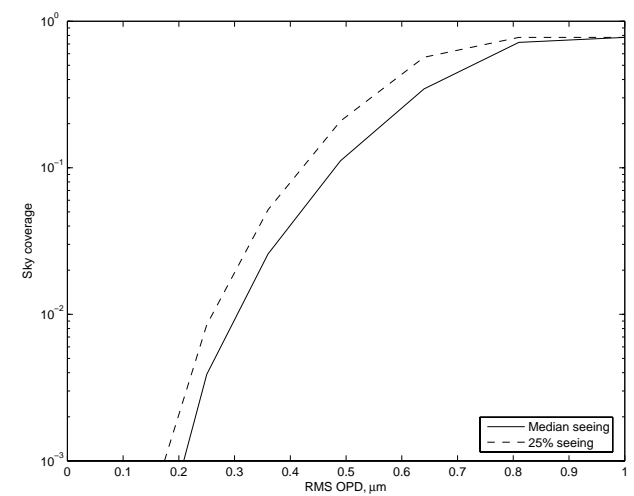

Figure 13. Sky coverage as a function of the residual RMS OPD for the NFIRAOS NGS AO system with either median or $25 \%$ seeing. These results are for the case of order $60 \times 60$ wavefront compensation and the E2V CCD-60 WFS detector parameters. The NFIRAOS implementation error budget of $101 \mathrm{~nm}$ RMS is not included.

conditions. ${ }^{\ddagger}$ Sky coverage improves by factors between $50 \%$ to $100 \%$ for $25 \%$ seeing at virtually all levels of performance.

Figures 14 and 15 plot sky coverage as a function of Strehl ratio and the enclosed energy within a square, 50 mas pixel. Results are presented for $\mathrm{J}, \mathrm{H}$, and $\mathrm{K}$ bands, either median (black) or $25 \%$ (red) seeing, and for these plots the NFIRAOS implementation error budget is included. Fully diffraction limited performance is achieved at small, but non-trivial, values of sky coverage. For example, Strehl ratios of about [0.10, 0.25, 0.45] are achieved in $[\mathrm{J}, \mathrm{H}, \mathrm{K}]$ bands with 1 per cent sky coverage for the case of median seeing. At 0.1 per cent sky coverage, the Strehls increase significantly to about $[0.3,0.5,0.65]$. The corresponding values with $25 \%$ seeing are $[0.15,0.35,0.55]$ and $[0.4,0.55,0.75]$. Finally, the enclosed energy results plotted in figure 15 are very nearly proportional to these Strehl ratios, indicating that most of the light lost from the diffraction-limited core of the PSF is scattered outside of a 50 mas pixel.

\section{ACKNOWLEDGMENTS}

The authors gratefully acknowledge the support of the TMT partner institutions. They are the Association of Canadian Universities for Research in Astronomy (ACURA), the California Institute of Technology and the

\footnotetext{
${ }^{\ddagger}$ Once again, the NFIRAOS implementation error budget is not included.
} 


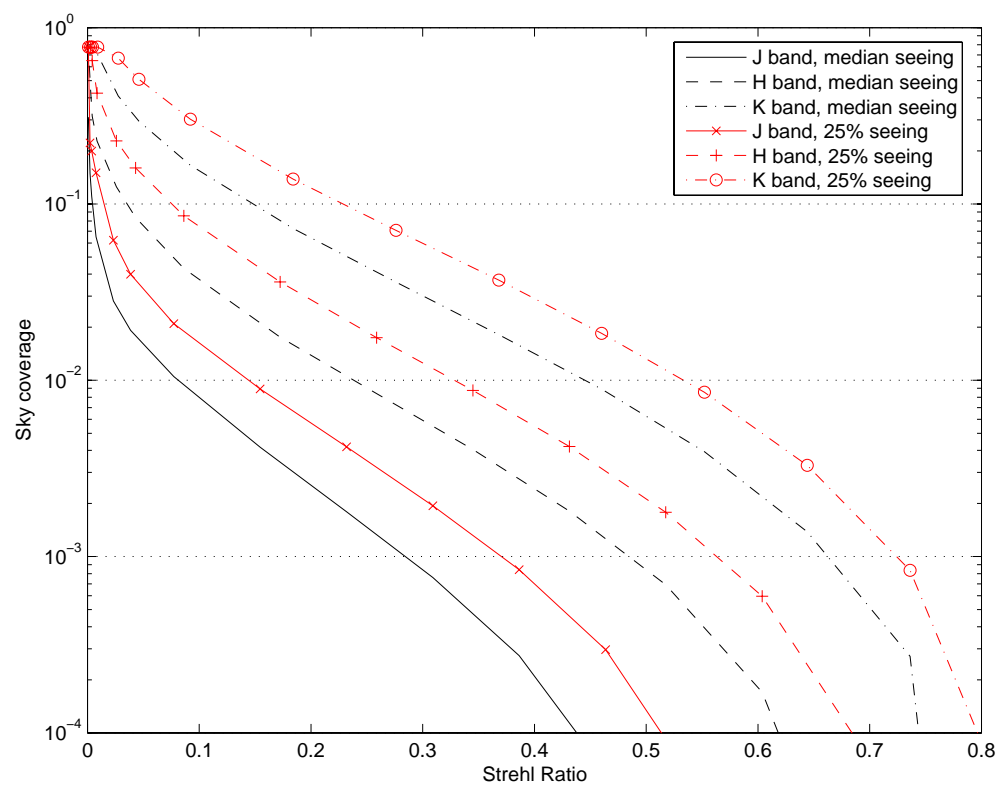

Figure 14. Sky coverage as a function of the Strehl ratio achieved in J, H, and $\mathrm{K}$ bands for the NFIRAOS NGS AO system with either median (black) or $25 \%$ (red) seeing. These results are for the case of $60 \times 60$ wavefront compensation and the E2V CCD-60 detector parameters. the NFIRAOS implementation error budget of $101 \mathrm{~nm}$ RMS is included.

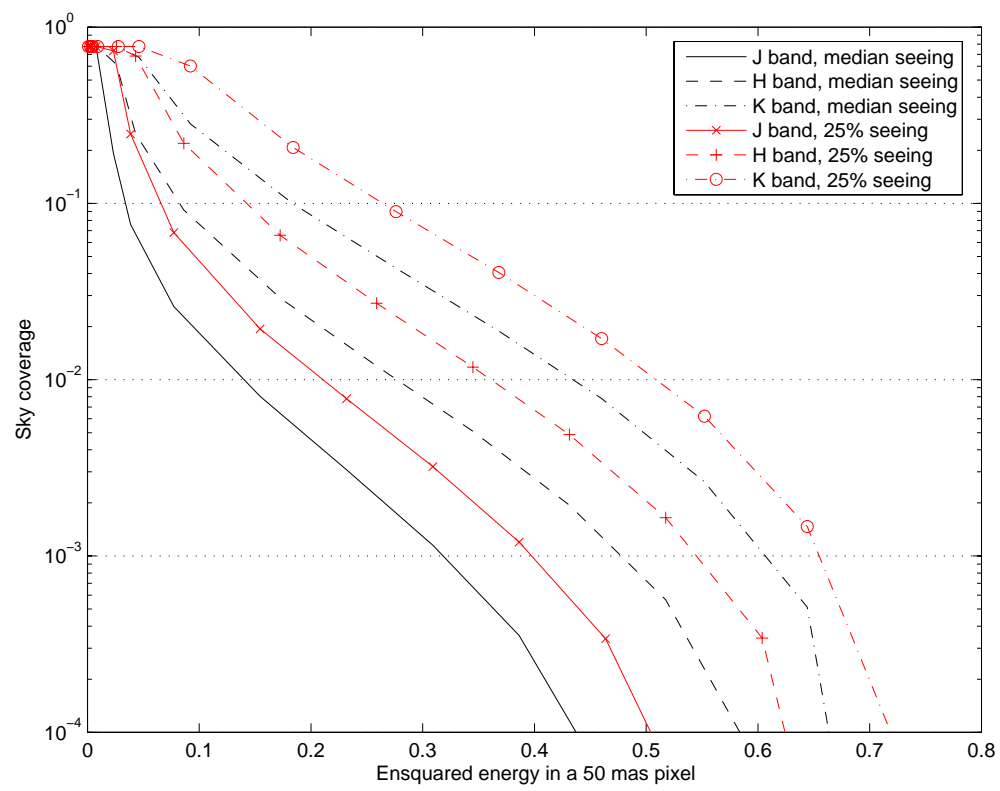

Figure 15. This figure is analogous to Figure 14 above, except that sky coverage is plotted as a function of the enclosed energy with a square, 50 mas pixel. 
University of California. This work was supported as well by the Gordon and Betty Moore Foundation, the Canada Foundation for Innovation, the Ontario Ministry of Research and Innovation, the National Research Council of Canada, the Natural Sciences and Engineering Research Council of Canada, the British Columbia Knowledge Development Fund, the Association of Universities for Research in Astronomy (AURA) and the U.S. National Science Foundation.

Additionally, Sean Adkins and Paul Jordan provided the information on the expected performance of future high-speed CCD arrays, and Jean-Pierre Véran assisted with the comparision of discrete- and continuous-time servo models.

\section{REFERENCES}

1. Science-Based Requirements Document (SRD) v16.4, TMT.PSC.DRD.05.001.CCR16, http://www.tmt.org:8080/docushare/dsweb/Get/Document-319/SRD v16 4.pdf.

2. EAP Final Report - June 27,2007, TMT.PMO.COR.07.029.REL01, http://www.tmt.org:8080/docushare/dsweb/Get/Document-8793/EAP Report final 62707.doc.

3. B. L. Ellerbroek and D. W. Tyler, "Adaptive Optics Sky Coverage Calculations for the Gemini-North Telescope," PASP, 110:165, 1998

4. Sean Adkins, personal communication.

5. Paul Jordan, personal communication.

6. Polar Coordinate CCD array for LGS AO, TMT.AOS.COR.06.008.DRF01, http://www.tmt.org:8080/docushare/dsweb/Get/Document-5218/TMT Polar Coordinate CCD Development Revised.doc.

7. L. Jolissait, J,-P. Véran, and R. Conan, "Analytical modeling of adaptive optics: the foundations of the phase spatial power spectrum approach," JOSA A 23:383, 2006.

8. Brent L. Ellerbroek, "Linear systems modeling of adaptive optics in the spatial-frequency domain," JOSA A, 22:310, 2005.

9. Glenn A. Tyler and David L. Fried, "Image-position error associated with a quadrant detector," JOSA, 72:804, 1982.

10. John N. Bahcall and Raymond M. Soneira, "Comparisons of a Standard Galaxy Model with Stellar Observations in Five Fields," ApJS, 55:67, 1984.

11. J.-F. LeBorgne et. al, "STELIB: a library of stellar spectra at R 2000," A \& A, 402, 433.

12. Thirty Meter Telescope Construction Proposal, TMT.PMO.MGT.07.009.REL02, http://www.tmt.org:8080/docushare/dsweb/Get/Document-8416/Proposal.pdf.

13. TMT Site Qualification Report - 2007-06-27 (Final Release), TMT.SIT.TEC.07.009.REL03, http://www.tmt.org:8080/docushare/dsweb/Get/Document-8467/TMT.SiteQualificationsReport.07-0630.pdf.

14. Gemini Mauna Kea sky background model, http://www.gemini.edu/sciops/ObsProcess/obsConstraints/ocSkyBackground.html.

15. Observatory Requirements Document (ORD), TMT.SEN.DRD.05.001.CCR14, http://www.tmt.org:8080/docushare/dsweb/Get/Document-2688/ORD-CCR14.pdf.

16. Jean-Pierre Véran, personal communication. 